International Journal of Clinical Therapeutics and Diagnosis (IJCTD)

ISSN 2332-2926

\title{
Updates in Vaccines, Viral Therapy and Non-Lymphocytic Immunotherapy for Glioblastoma
}

\author{
Review Article
}

Wu A ${ }^{1 *}$, Jackson $\mathrm{C}^{2}$, Lim $\mathrm{M}^{2}$

1 Johns Hopkins University School of Medicine, Baltimore, MD, USA.

2 Department of Neurosurgery, Johns Hopkins Hospital, Baltimore, MD, USA.

\section{Abstract}

A key focus of glioblastoma (GBM) management presently revolves around the cancer's ability to evade and suppress anti-tumor immune response. Therefore, cancer immunotherapy is a critical area in development and investigation with numerous ongoing clinical trials and animal studies. Ranging from vaccines containing tumor-associated antigens or antigen-presenting dendritic cells to viral therapies and immune checkpoint inhibitor antibodies, immunotherapy offers novel therapeutic avenues for GBM as well as other solid and hematogenous cancers. This review provides an overview and update on various significant immunotherapy developments in addition to introducing novel targets for cancer treatment.

Keywords: Glioblastoma; Immunotherapy; Vaccines; Oncolytic Viral Therapy; Immune Checkpoint Inhibitor; Immune System.

\section{Introduction}

Glioblastoma(GBM), the most common primary brain tumor in adults, is still a formidable disease with median survival of 1416 months despite current standard of care involving surgical resection with adjuvant chemoradiation therapy. [1, 2]. In recent years, immunotherapy has become a new avenue for GBM treatment with the discovery that the microenvironment created by the presence of GBM suppresses the normal immune system function, thereby allowing cancers to evade cytotoxic mechanisms, and that reversal of the immunosuppression may limit progression of the tumor [3]. According to the Cancer Research Institute, there are currently at least 27 ongoing clinical trials in brain cancer immunotherapy, including cancer vaccines, checkpoint inhibitors, oncolytic virus therapies, adoptive immune cell therapy, adjuvant therapies as well as monoclonal antibodies [4]. Clinicaltrials.gov also lists 68 completed and ongoing glioblastoma clinical trials in various stages of recruitment and completion for a variety of vaccines and immunotherapy treatments.

GBM's evasion of the immune system is multi-faceted.
The involvement of CD4+ and CD8+ lymphocytes in the pathogenesis of GBM has been studied in detail. In recent works, tumor-associated macrophages (TAMs) have also been found to be intimately associated with the microenvironment of gliomas, which promotes tumor progression [5]. In particular, M2-type macrophages, which support tumor growth through increasing immunosuppressive factors, such as TGF- $\beta$, block the activation of various immune cells and suppress including suppression of activated CD8+ T-cells, therefore preventing anti-tumor functions $[5,6,7,8]$. Neutrophils and granulocytes also play a role in the tumor microenvironment that manipulates the immune response.

Investigation and development of immune based therapies targeting separate components of the immunosuppressive glioma microenvironment would be a useful addition to treatment options for halting the progression of GBM. We seek to provide a summary and update on recent advancements in GBM immunotherapy, namely of viral therapies, checkpoint inhibitors and vaccine formulations, with an additional lens on recent nonlymphocyte targets.

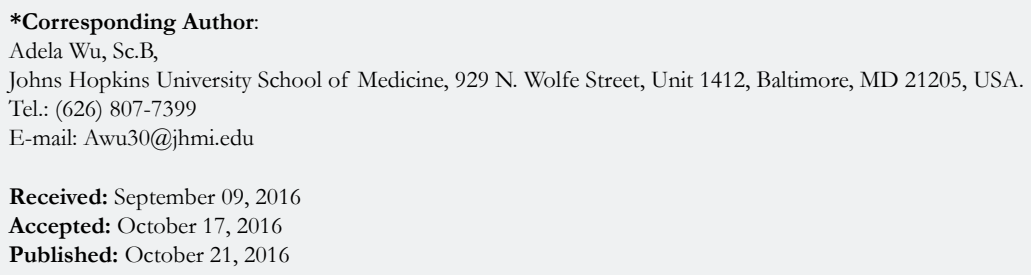

Copyright: $\mathbf{W u} \mathbf{A}^{\circ}$ 2016. This is an open-access article distributed under the terms of the Creative Commons Attribution License, which permits unrestricted use, distribution and reproduction in any medium, provided the original author and source are credited. 


\section{Immunosuppressive Tumor Microenvironment and the Development of Immunotherapy}

Since the discovery of the immune system's role in neoplastic processes, immunotherapy is now poised at the cutting edge for cancer treatment [9]. From research demonstrating the significant clinical benefit of trastuzumab as immunotherapy for metastatic breast cancer, the FDA's approval of sipuleucel-T [Provenge] for prostate cancer treatment in 2010 and of the anti-CTLA-4 monoclonal antibody ipilimumab for late-stage melanoma in 2011, the field of oncological immunotherapy has picked up rapid pace $[10,11]$. With regards to malignant gliomas, a variety of immune modalities have been extensively studied and considered, including harnessing stimulatory cytokines, administering antitumor antibodies or serotherapy, and immunogenic vaccines [12].

Glioblastoma(GBM) in particular still carries a dismal prognosis despite the current standard of care including surgery, radiotherapy, and adjuvant temozolomide chemotherapy [13]. One of the biggest difficulties in managing GBM involves various factors contributing to its ability to evade the tumor-targeting immune responses. To begin with, the central nervous system is uniquely buffered by the blood-brain barrier, which establishes a selective filter for specific T-cells [14]. Additionally, in conditions of chronic inflammation or cancer, many T-cells are in a state of exhaustion, characterized as changes in cytokine signaling and metabolism that prevent T-cell proliferation and effector capabilities. Furthermore, there tends to be an increase in the expression of immunosuppressive FoxP3+ regulatory lymphocytes which inhibit the activities of the effector FoxP3- T cells. [3, 14, 15]. If the immune system could be returned to its original functionality through immunotherapy, whether it be increases in CD3+ and CD8+ T-lymphocyte infiltration or an increase in ratio of effector $T$ cells to T regulatory cells, augmentation of granulocytic activity can translate into prolonged survival for GBM patients [16].

\section{Vaccines}

\section{Tumor-Associated and Tumor-Specific Antigens}

Tumor-infiltrating lymphocytes could recognize and target tumor-specific antigens [TSA] and tumor-associated antigens [TAA] as part of the body's immune response similar to the body's lymphocytic response to viral illnesses. Some of the first discoveries of the role of TAA and TSA came from studies of melanoma. Through utilizing cytotoxic T-lymphocyte clones against autologous melanoma, Knuth et al., were able to identify three unique antigens expressed on the tumor cells of melanoma which helped shine light on a potential mechanism of tumor's evasion of the immune system. The mechanism of antigen loss prevented recognition of tumor antigen and targeting by cytotoxic T cells. [17].

Melanoma also served as the model for the discovery of the first TAA, MAGE-1, in 1991 [18] as well as the ability to create cytolytic T-cell clones that could target the antigen. Other melanoma antigenic targets that have since been recognized as potential therapeutic targets include gp100 and MAGE-3 [19, 20].

In the specific context of glioma, TSA such as mutated EGFRvIII are potentially excellent candidates for immunotherapy because of their specificity and inherent immunogenicity due to their foreign nature. Antigenic vaccines serve to deliver antigens to allow native T-cells to be primed to recognize TSA and thereby generate an immune response to the tumor [21, 22]. One such vaccine RINTEGA (rindopepimut) enhances immune response against EGFRvIIIfor GBM management [23]. In numerous animal models, vaccination with rindopepimut elicited antibody production and targeted binding of EGFRvIII receptors [24]. Unfortunately, Celldex Therapeutics, which manufactures Rindopepimut, recently announced the failure of the vaccine to improve EGFRvIII-positive GBM patients' overall survival compared to temozolomide administration and the control group in the Phase III ACT IV study, discontinued in March 2016 [25]. Their Phase II studies - ACTIVATE, ACT II, ACT III- showed that Rindopepimut achieved median overall survival times ranging from 24.3-24.6 months in patients with EGFRvIII-expressing GBM. However, the Phase III ACT IV study demonstrated that the control arm performed better than anticipated with the comparison of median overall survival being 20.4 months (Rindopepimut) and 21.1 months (control).

Clinical trials on three other TAAs-interleukin-13 receptor alpha 2, EphA2, have also been studied in the survival of pediatric patients with glioblastomas and brainstem gliomas [26]. Patients with GBM tumors that expressed any of the following TAAgp100, MAGE-1, HER2, TRP-2, AIM-2, IL13Ra2, were enrolled in a study and given an autologous dendritic cell vaccine with the TAAs, resulting in improved survival times depending on the TAA and prolonged progression-free survival in patients with newlydiagnosed GBM [27]. Furthermore, Gliovac, a prototype vaccine comprising of both autologous GBM TAAs and allogeneic GBM TAAs as well as GM-CSF, in combination with standard of care treatment resulted in 100\% 6-month survival for patients compared to $33 \%$ 6-month survival in control patients [28].

\section{Cell-Based Therapy and Vaccines}

Given the key roles and interactions cytokines and tumor cells themselves play in sustaining the immunosuppressive tumor microenvironment in cancer states, a natural avenue for oncologic immunotherapy to turn towards was cell-based and cytokinesecreting tumor cell vaccines.

Historically, in the 1970s, Trouillas et al., showed that high-grade astrocytoma patients receiving both radiation therapy and an autologous tumor cell vaccine had prolonged survival compared to those who only received radiation [29]. It has been shown that cytokine activity leads to potent immune system activity against neoplasms. Iwadate et al., showed that introduction of IL-2 with cytokine gene therapy in the microenvironment of brain tumor led to upregulation of tumor-infiltrating CD4+ and CD8+ T-cells [30]. Dranoff et al., created a series of different viruses and vaccines targeting different molecules involved in tumorigenicity which showed that tumor cells containing granulocyte-macrophage colony-stimulating factor [GM-CSF] promoted immunity against tumor cells through recruitment of dendritic cells, which then actively phagocytose and present tumor cells as antigens to other effector immune cells [31].

Since then, GM-CSF vaccines have been studied in numerous clinical trials for different cancers, including renal cell carcinoma, 
non-small-cell lung cancer, breast cancer, and pancreatic cancer $[32,33,34,35,36]$. Specifically in GBM, the combination of GM-CSF with IFN-gamma immunization improved survival in a glioblastoma murine model [37]. Another study showed that a combined vaccination of tumor cells transduced with GMCSF and the T-cell co-stimulatory protein, B7-2, successfully suppressed tumor cell growth at distant sites [38]. A recent Phase I clinical trial on GBM also demonstrated that the combination of GM-CSF with a vaccine containing multiple TAAs resulted in progression-free survival of $74 \%$ of patients at 6 months and $31 \%$ at 9 months [39] Whether as stand-alone therapy or as a combination avenue, GM-CSF and other cell-based therapies could hold substantial promise for cancer and GBM management.

\section{Dendritic Cell Vaccines}

Central to the immune system response are antigen-presenting cells that interact with T-cells, which can then target tumor cells and perform effector functions. Dendritic cells [DC] are especially suited for antigen presentation as they congregate in areas of high antigen exposure and rapidly upregulate the necessary cytokines and signaling to contact naïve T-cells [40]. DC could also present tumor antigens, establishing these cells as promising potential targets of immunotherapy [41]. In addition, DC play a significant role in combating the immunosuppressive tumor microenvironment. When tumor cell lysates were hitched to DCs, there was a subsequent increase in phagocytosis and humoral immunity response as well as improved long-term survival in a glioma model [42]. DCs also elicit more tumor-infiltrating T-cells and anti-tumor activity in a GBM clinical trial [43].

DC-containing cancer vaccines have featured prominently in animal studies and human clinical trials since the first study on B-cell lymphoma in mice in 1996 [44]. Various cancers have been studied with DC vaccine treatment, including renal cell carcinoma, melanoma, and sarcomas [45, 46, 47]. The first FDA-approved immunotherapeutic was the anti-tumor vaccine, sipuleucel-T, which includes DCs in its formulation, and it has been shown to improve survival and patient outcomes in advanced prostate cancer $[48,49]$.

DC vaccination has been found to suppress glioma and GBM growth through a variety of mechanisms, leading to improved clinical outcomes for patients. DC vaccines in combination with anti-angiogenesis molecules suppressed both angiogenesis processes as well as activity of glioma stem-cell-like cells [50]. The addition of DC vaccination also elicited a shift from regulatory T-cells to effector T-cells and a strong anti-tumor response, thereby improving glioma-bearing mice's survival by 300\% [51]. Chang et al., followed GBM patients who were administered autologous DC-tumor vaccinations, which resulted in tumor regression as well as enhanced tumor-infiltrating T-cell counts and prolonged median and long-term survival [52]. Recently, a clinical trial on recurrent GBM tumors demonstrated that the combination of temozolomide and a vaccine that contained DCs and autologous tumor cells resulted in prolonged progressionfree survival in a number of the patients [53].

\section{Adoptive $T$ cell and Chimeric Antigen Receptor Immunotherapy}

Adoptive T-cell therapy (ACT) involves isolating tumor-infiltrating lymphocytes, expanding them in vitro and then transferring the cells back to the patient. The infused adoptive cytotoxic T-cells will then commence eliminating neoplasms [54]. Everson et al studied the effect of adoptive T cells targeting NY-ESO-1 in a murine model for human glioblastoma and found that NY-ESO-1 promoted a glioma-specific anti-tumor response and conferred survival benefit [55].

The development of chimeric antigen receptors (CARs)made it more efficient to create adoptive T-cells specific for TAAs. CARs are comprised of an extracellular portion that recognizes antigen and an intracellular portion that involves signaling domains, which leads to improved lymphocyte proliferation and anti-apoptotic molecule generation as well as enhanced resistance against the immunosuppressive tumor microenvironment with the addition of CD28 to the CAR [56, 57]. Chow et al., used CAR constructs to generate T-cells specifically against erythropoietin-producing hepatocellular carcinoma A1 [EphA2], which is overexpressed on surfaces of malignant glioma cells [58]. An alternative to CARs, adoptive $\mathrm{T}$ cells with bispecific T-cell engager (BiTE), was successfully used in a leukemia mouse model to show that T-cells could be induced to recognize and perform significant antitumor activity against CD19+ cell lines, leading to complete cancer remission [92].

Adoptive $\mathrm{T}$ cell therapy and CARs have also been adapted for GBM management. In vitro and in vivo animal studies demonstrated strong anti-glioma activity and disease regression. In addition, the first clinical trial of intracranially-administered IL13[E13Y]zetakine $\mathrm{CD} 8[+]$ CTL targeting IL13R $\alpha 2$ for recurrent GBM demonstrated that $2 / 3$ patients experienced temporary antiglioma responses [59]. Current trials include an EGFRvIIItargeted CAR T-cell approach in recurrent GBM tumors at the University of Pennsylvania [93].

\section{Immune Checkpoint Receptor Inhibitors}

Immune checkpoint receptors are well-known entities within the field of cancer immunotherapy at this point. Checkpoint receptors promote immunosuppression through the orchestration of antigen-presenting cells and effector and helper cells [60]. In a normal physiologic state, checkpoint receptors are helpful in that they prevent auto-immunity. However, neoplasms could exploit immune checkpoint receptors to evade the immune response. PD1, expressed on T-cells, interacts with its ligands PD-L1 and PDL2 and contributes to both CD4+ and CD8+ T-cell exhaustion [61]. CTLA-4 has been shown to decrease T-cell activation by establishing a competing site to bind on antigen-presenting cells. Therefore, antibodies that serve as immune checkpoint inhibitors could prevent T-cell suppression and exhaustion and eliminate the tumor microenvironment's immune resistance [62].

Historically, the immune checkpoints PD-1 and CTLA-4 have been the most studied. Following successful trials in animals, the 
first human clinical trial of anti-PD-1 in patients with a variety of solid neoplasms demonstrated that disease regression was possible with administration of anti-PD-1 monoclonal antibodies [MDX1106] [63]. The administration of ipilimumab, an anti-CTLA-4 antibody, to metastatic melanoma and ovarian cancer patients who had already received dendritic cell vaccines that enhanced antigen presentation led to promising results as well [64]. Recent clinical trials on anti-PD-1 and anti-CTLA-4 have included those for lung cancer and metastatic melanoma $[65,66]$

Immunotherapy centered on immune checkpoint receptors in combination with existing treatment strategies has been significant for GBM management. In murine models of GBM, the combination of anti-PD-1 and radiotherapy showed improved survival [67]. In a glioblastoma study, mice were treated with 4-1BB agonist antibodies, anti-CTLA-4 antibodies, and radiation therapy. The combination therapy resulted in increased survival times of at least 50\% long-term disease-free survival as well as greater counts of tumor-infiltrating lymphocytes [68]. Novel immune checkpoint receptor inhibitors have also emerged, such as anti-TIM-3. Combined triple therapy with anti-TIM-3, anti-PD-1, and stereotactic radiosurgery resulted in $100 \%$ overall survival in a murine GBM model with concurrent increases in anti-tumor T-cell infiltration and immune response [69]. Another monoclonal antibody directed against GITR, a molecule expressed on regulatory T-cells and involved in costimulatory signaling, also significantly improved survival rates in a glioma murine model when it was administered in conjunction with stereotactic radiosurgery [94]. With the variety of immune checkpoint inhibitor targets expressed within GBM, there is much more to be investigated in future clinical trials of GBM immunotherapy management in particular.

\section{Viral Therapies}

Oncolytic virus immunotherapy is a recent and upcoming avenue to combat cancers through utilizing viruses that could specifically replicate within and destroy cancer cells in addition to carrying antitumor factors. Talimogenelaherparepvec [T-VEC] just became the first oncolytic virus therapy to be FDA-approved in the United States and Europe [84]. T-VEC is based on the herpes simplex virus type-1 structure and incorporates GM-CSF for promotion of antitumor response. From an earlier phase II trial utilizing the GM-CSF-expressing oncolytic herpes virus, named Oncovex in the trial, the injection increased antitumor response and diminished immunosuppressive Tregs and myeloid-derived suppressive cells in melanoma lesions [85]. In a phase III trial for patients with advanced melanoma, T-VEC administration resulted in prolonged overall survival as well as improved duration of response compared to administration of solely GM-CSF [86]. T-VEC also led to regression of melanoma lesion size [87].

HSV-1 also figures significantly in research and clinical trials targeted towards glioblastoma. M032 is a synthetic HSV-1 oncolytic virus therapy that expresses IL-12, which itself has antitumor and antiangiogenic effects. Thus far, M032 has been deemed safe for use in animal models [88].

In addition, viral peptides also could serve as targets for immunotherapy. Cytomegalovirus (CMV) peptides have been identified as glioma-associated antigens [89]. Schuessler et al., utilized autologous T-cell therapy directed towards CMV antigens in glioblastoma, finding that $40 \%$ of patients receiving the therapy were progression-free during the follow-up period and that patient response was associated with the genetic expression profiles of the individual tumors [90]. There is also currently an ongoing clinical trial at Baylor College of Medicine for administration of T-cells targeting CMV antigens in GBM [91].

\section{Non-Lymphocytic Cells and Their Potential for Future Immunotherapeutics}

Macrophages serve a vital purpose in the immune system by presenting antigens to lymphocytes and phagocytosing debris in addition to regulating the immune and inflammatory response [3]. There are two broad categories of macrophages based on their surrounding environment-M1, which promotes inflammatory cytokines, and M2, which promotes anti-inflammatory signaling and immunosuppression and includes tumor-associated macrophages [TAM]. TAMs are associated with tumor and disease progression and poor clinical outcomes [70]. A proposed mechanism of the pro-tumor growth influence of M2-type macrophages in the tumor microenvironment has been the secretion of chemokine periostin by glioblastoma stem cells [GSC], which leads to the recruitment and maintenance of M2-type TAMs as well as other monocyte derivatives [71]. TAMs even promote cancer metastases by altering the tumor microenvironment through factors, such as tumor-derived transforming growth factor and macrophage migration inhibitor factor [MIF] [72]. In addition, macrophages could be duped by certain cancer cells that expressed CD47, a molecule that inhibited immune cells' phagocytic activity [73]. Overall, presence of M2-type tumor-infiltrating macrophages is positively correlated with the WHO grade of glioma, thereby associated with tumor growth and poor prognosis [74]. On the other hand, it has been shown that M1-type macrophages may represent a better therapeutic outcome for glioma patients [74].

Macrophages could be harnessed in cancer immunotherapy to combat tumor cells. TAMs that expressed TNF receptors on their surfaces were heavily involved in promoting glioma growth. However, blockade of TNF led to glioma tumor cell elimination and apoptosis [75]. Immunotherapy and induction of IL-15 also can protect $\mathrm{V} \alpha 24$-invariant NKT cells, which themselves have the property of inhibiting tumorigenesis by targeting TAMs [76].

Tumor-associated neutrophils contribute to cancer immunosuppression through dampening CD8+ T-cell activity and promoting tumorigenesis [72] For example, in hepatocellular cancer, tumor-infiltrating neutrophils that expressed PD-L1 suppressed T-cell activity and proliferation [77]. Of note, the ratio of neutrophils to lymphocytes has been correlated with poor clinical outcomes with higher neutrophil counts in colorectal cancer and glioblastoma [78, 79]. GBM tumors contained a significantly greater count of infiltrating neutrophils than even other types of glioma tumors [80]. In addition, CXCR2 is a tumorigenic IL-8 receptor that promotes neutrophil migration in an inflammatory environment, and it has been demonstrated that a high level of CXCR2 expression within gliomas was associated with likelihood of recurrence. Furthermore, inhibiting CXCR2 activity lowered glioma cell migration and proliferation [81] However, in another level of complexity in neutrophils' role in the tumor microenvironment, Graf et al., showed that neutrophils played a key role in an IL 6-induced anti-glioma response, and 
that mice that were neutropenic could not reject glioma tumors [82]. Another role neutrophils play in cancer immunotherapy is as an innovative vehicle for delivery of anti-tumor monoclonal antibodies. Nanoparticles programmed to hijack neutrophils recruited by tumor shuttle a monoclonal antibody targeting the gp75 antigen in melanoma, leading to tumor growth suppression and improved survival in a murine model [83]. New strategies employing neutrophils for GBM management are still ongoing, with manipulating CXCR2 and other similar receptors through immunotherapy.

\section{Conclusion and Future Directions}

Cancer treatment has made significant strides with the introduction and implementation of various immunotherapy modalities, including cell-based therapies, oncolytic virus therapies, immune checkpoint inhibitors, and development of non-lymphocytetargeted therapeutics. This review gives a broad overview on both the rationale underlying and recent updates to immunotherapy for a variety of cancers with a focus on glioblastoma(GBM). GBM tumors generate a profoundly immunosuppressive tumor microenvironment that dampens effector T-cell response and upregulates inhibitory cytokines and signaling. To produce enhanced antitumor responses and prolong patient survival times, different immunotherapy strategies have been employed, from DC vaccines to novel oncolytic virus therapies. A trend in recent immunotherapy clinical trials for GBM and other cancers has been to investigate the efficacy and safety of combined therapies, such as immune checkpoint inhibitors in concurrence with radiotherapy or a DC vaccine containing autologous tumor cells in concurrence with temozolomide chemotherapy. Thus far, the clinical implications have been promising and have opened new routes for future research into different receptors and cells that could play pivotal roles in cancer immunotherapy for GBM.

\section{References}

[1]. Domingo-Musibay E, Galanis E (2015) What next for newly diagnosed glioblastoma?. Future Oncol. 11(24): 3273-83. Doi: 10.2217/fon.15.258.

[2]. Wilson TA, Karajannis MA, Harter DH (2014) Glioblastoma multiforme: State of the art and future therapeutics. SurgNeurol Int. 5: 64.

[3]. Razavi SM, Lee KE, Jin BE, Aujla PS, Gholamin S, et al., (2016) Immune Evasion Strategies of Glioblastoma. Frontiers of Surgery. 3: 11.

[4]. Immunotherapy for Brain Cancer (2016) Cancer Research Institute. http:// www.cancerresearch.org/cancer-immunotherapy/impacting-all-cancers/ brain-cancer

[5]. Kennedy BC, Showers CR, Anderson DE, Anderson L, Canoll P, et al., (2013) Tumor-Associated Macrophages in Glioma: Friend or Foe?. J Oncol.

[6]. Umemura N, Saio M, Suwa T, Kitoh Y, Bai J, et al., (2008) Tumor-infiltrating myeloid-derived suppressor cells are pleiotropic-inflamed monocytes/ macrophages that bear M1- and M2-type characteristics. J Leukoc Biol. 83(5): 1136-44.

[7]. Komohara Y, Ohnishi K, Kuratsu J, Takeya M (2008) Possible involvement of the M2-anti-inflammatory macrophage phenotype in growth of human glioma. J Pathol. 216(1): 15-24.

[8]. Wrzesinski SH, Wan YY, Flavell RA (2007) Transforming growth factorbeta and the immune response: implications for anticancer therapy. Clin Cancer Res. 13(18): 5262-70.

[9]. Roberts JA (1977) Immunotherapy in the treatment of cancer. Scott Med J. 22(4): 320-30.

[10]. Slamon DJ, Leyland-Jones B, Shak S, Fuchs H, Paton V, et al., (2001) Use of chemotherapy plus a monoclonal antibody against HER2 for metastatic breast cancer that overexpresses HER2. N Engl J Med. 344(11): 783-92.

[11]. Mansh M (2011) Ipilimumab and cancer immunotherapy: a new hope for advanced stage melanoma. Yale J Biol Med. 84(4): 381-9.

[12]. Parney IF, Hao C, Petruk KC (2000) Glioma immunology and immunotherapy. Neurosurgery. 46(4): 778-91
[13]. Weller M, van den Bent M, Hopkins K, Tonn JC, Stupp R, et al., (2014) EANO guideline for the diagnosis and treatment of anaplastic gliomas and glioblastoma. Lancet Oncol. 15(9): e395-403.

[14]. Haque A, Banik NL, Ray SK (2011) Molecular alterations in glioblastoma: potential targets for immunotherapy. Prog Mol Biol Transl Sci. 98: 187-234.

[15]. Wherry EJ, Kurachi M (2015) Molecular and cellular insights into T cell exhaustion. Nat Rev Immunol. 15(8): 486-99.

[16]. Kmiecik J, Poli A, Brons NH, Waha A, Eide GE, et al., (2013) Elevated $\mathrm{CD} 3$ + and $\mathrm{CD} 8$ + tumor-infiltrating immune cells correlate with prolonged survival in glioblastoma patients despite integrated immunosuppressive mechanisms in the tumor microenvironment and at the systemic level. J Neuroimmunol. 264(1-2): 71-83.

[17]. Knuth A, Wölfel T, Klehmann E, Boon T, Meyer zum Büschenfelde KH (1989) Cytolytic T-cell clones against an autologous human melanoma: Specificity study and definition of three antigens by immunoselection. ProcNatl Acad Sci. 86: 2804-8.

[18]. van der Bruggen P, Traversari C, Chomez P, Lurquin C, De Plaen E, et al., (1991) A gene encoding an antigen recognized by cytolytic T lymphocytes on a human melanoma. Science. 254(5038): 1643-7.

[19]. Kawakami Y, Eliyahu S, Delgado CH, Robbins PF, Sakaguchi K, et al., (1994) Identification of a human melanoma antigen recognized by tumorinfiltrating lymphocytes associated with in vivo tumor rejection. Proc Natl Acad Sci. 91(14): 6458-62.

[20]. Chaux P, Vantomme V, Stroobant V, Thielemans K, Corthals J, et al., (1999) Identification of MAGE-3 epitopes presented by HLA-DR molecules to CD4+ T lymphocytes. J Exp Med. 189(5): 767-78.

[21]. Sugawa N, Ekstrand AJ, James CD, Collins VP (1990) Identical splicing of aberrant epidermal growth factor receptor transcripts from amplified rearranged genes in human glioblastomas. Proc Natl Acad Sci. 87(21): 8602-6.

[22]. Ilyas S, Yang JC (2015) Landscape of Tumor Antigens in T cell Immunotherapy. J Immunol. 195(11): 5117-22.

[23]. Paff M, Alexandru-Abrams D, Hsu FP, Bota DA (2014) The evolution of the EGFRvIII (rindopepimut) immunotherapy for glioblastoma multiforme patients. Hum Vaccin Immunother. 10(11): 3322-31.

[24]. Wikstrand CJ, Stanley SD, Humphrey PA, Pegram CN, Archer GE, et al., (1993) Investigation of a synthetic peptide as immunogen for a variant epidermal growth factor receptor associated with gliomas. J Neuroimmunol. 46(1-2): 165-73.

[25]. RINTEGA (rindopepimut) - A Phase 3 Immunotherapy Targeting EGFRvIII-expressing Glioblastoma (GBM). (2016) Celldex Therapeutics.

[26]. Pollack IF, Jakacki RI, Butterfield LH, Hamilton RL, Panigrahy A, et al., (2014) Antigen-specific immune responses and clinical outcome after vaccination with glioma-associated antigen peptides and polyinosinic-polycytidylic acid stabilized by lysine and carboxymethylcellulose in children with newly diagnosed malignant brainstem and nonbrainstemgliomas. J ClinOncol. 32(19): 2050-8.

[27]. Phuphanich S, Wheeler CJ, Rudnick JD, Mazer M, Wang H, et al., (2013) Phase I trial of a multi-epitope-pulsed dendritic cell vaccine for patients with newly-diagnosed glioblastoma. Cancer Immunol Immunother. 62(1): 125-35.

[28]. Schijns VE, Pretto C, Devillers L, Pierre D, Hofman FM, et al., (2015) First clinical results of a personalized immunotherapeutic vaccine against recurrent, incompletely resected, treatment-resistant glioblastoma multiforme (GBM) tumors, based on combined allo- and auto-immune tumor reactivity. Vaccine. 33(23): 2690-6.

[29]. Trouillas P (1973) Immunology and immunotherapy of cerebral tumors: Current status. Rev Neurol. 128(1): 23-38.

[30]. Iwadate Y, Yamaura A, Sato Y, Sakiyama S, Tagawa M (2001) Induction of immunity in peripheral tissues combined with intracerebral transplantation of interleukin-2-producing cells eliminates established brain tumors. Cancer Res. 61(24): 8769-74.

[31]. Dranoff G, Jaffee E, Lazenby A, Golumbek P, Levitsky H et al., (1993) Vaccination with irradiated tumor cells engineered to secrete murine granulocyte-macrophage colony-stimulating factor stimulates potent, specific, and long-lasting anti-tumor immunity. ProcNatlAcad Sci. 90(8): 3539-43.

[32]. Simons JW, Jaffee EM, Weber CE, Levitsky HI, Nelson WG, et al., (1997) Bioactivity of autologous irradiated renal cell carcinoma vaccines generated by ex vivo granulocyte-macrophage colony-stimulating factor gene transfer. Cancer Res. 57(8): 1537-46.

[33]. Salgia R, Lynch T, Skarin A, Lucca J, Lynch C, et al., (2003) Vaccination with irradiated autologous tumor cells engineered to secrete granulocytemacrophage colony-stimulating factor augments antitumor immunity in some patients with metastatic non-small-cell lung carcinoma. J ClinOncol. 21(4): 624-30.

[34]. Nemunaitis J, Jahan T, Ross H, Sterman D, Richards D, et al., (2006) Phase I/II trial of autologous tumor mixed with an allogeneic GVAX vaccine in advanced-stage non-small-cell lung cancer. Cancer Gene Ther. 13(6): 555-62. 
[35]. Laheru D, Lutz E, Burke J, Biedrzycki B, Solt S, et al., (2008) Allogeneic granulocyte macrophage colony-stimulating factor-secreting tumor immunotherapy alone or in sequence with cyclophosphamide for metastatic pancreatic cancer: a pilot study of safety, feasibility, and immune activation. Clin Cancer Res. 14(5): 1455-63.

[36]. Emens LA, Asquith JM, Leatherman JM, Kobrin BJ, Petrik S, et al., (2009) Timed sequential treatment with cyclophosphamide, doxorubicin, and an allogeneic granulocyte-macrophage colony-stimulating factor-secreting breast tumor vaccine: a chemotherapy dose-ranging factorial study of safety and immune activation. J ClinOncol. 27(35): 5911-18.

[37]. Smith KE, Janelidze S, Visse E, Badn W, Salford L, et al., (2007) Synergism between GM-CSF and IFNgamma: enhanced immunotherapy in mice with glioma. Int J Cancer. 120(1): 75-80.

[38]. Parney IF, Petruk KC, Zhang C, Farr-Jones M, Sykes DB, et al., (1997) Granulocyte-macrophage colony-stimulating factor and B7-2 combination immunogene therapy in an allogeneic Hu-PBL-SCID/beige mouse-human glioblastoma multiforme model. Hum Gene Ther. 10; 8(9): 1073-85.

[39]. Rampling R, Peoples S, Mulholland PJ, James A, Al-Salihi O, et al. (2016) A Cancer Research UK First Time in Human Phase I Trial of IMA950 (Novel Multi Peptide Therapeutic Vaccine) in Patients with Newly Diagnosed Glioblastoma. Clin Cancer Res. 22(19): 4776-4785.

[40]. Grabbe S, Beissert S, Schwarz T, Granstein RD, et al., (1995) Dendritic cells as initiators of tumor immune responses: a possible strategy for tumor immunotherapy? Immunol Today. 16(3): 117-21.

[41]. Huang AY, Golumbek P, Ahmadzadeh M, Jaffee E, Pardoll D, et al., (1994) Role of bone marrow-derived cells in presenting MHC class I-restricted tumor antigens. Science. 264(5161): 961-5.

[42]. Mineharu Y, King GD, Muhammad AK, Bannykh S, Kroeger KM, et al. (2011) Engineering the brain tumor microenvironment enhances the efficacy of dendritic cell vaccination: implications for clinical trial design. Clin Cancer Res. 17(14): 4705-18.

[43]. Yamanaka R, Abe T, Yajima N, Tsuchiya N, Homma J, et al. (2003) Vaccination of recurrent glioma patients with tumor lysate-pulsed dendritic cells elicits immune responses: results of a clinical phase I/II trial. Br J Cancer. 89(7): 1172-9.

[44]. Bohlen H, Thielemanns K, Tesch H, Engert A, Wolf HJ, et al. (1996) Idiotype vaccination strategies against a murine B-cell lymphoma: dendritic cells loaded with idiotype and bispecificidiotype $\mathrm{x}$ anti-class II antibodies can protect against tumor growth. Cytokines MolTher. 2(4): 231-8.

[45]. Märten A, Flieger D, Renoth S, Weineck S, Albers P, et al., (2002) Therapeutic vaccination against metastatic renal cell carcinoma by autologous dendritic cells: preclinical results and outcome of a first clinical phase I/II trial. Cancer ImmunolImmunother. 51(11-12): 637-44.

[46]. Hersey P, Menzies SW, Halliday GM, Nguyen T, Farrelly ML, et al. (2004) Phase I/II study of treatment with dendritic cell vaccines in patients with disseminated melanoma. Cancer ImmunolImmunother. 53(2): 125-34.

[47]. Dillman RO, Selvan SR, Schiltz PM, McClay EF, Barth NM, et al. (2004) Phase I/II trial of autologous tumor cell-line derived vaccines for recurrent or metastatic sarcomas. Cancer Biother Radiopharm. 19(5): 581-8.

[48]. Kantoff PW, Higano CS, Shore ND, Berger ER, Small EJ, et al., (2010) Sipuleucel-T immunotherapy for castration-resistant prostate cancer. N Engl J Med. 363(5): 411-22.

[49]. Higano CS, Schellhammer PF, Small EJ, Burch PA, Nemunaitis J, et al. (2009) Integrated data from 2 randomized, double-blind, placebo-controlled, phase 3 trials of active cellular immunotherapy with sipuleucel- $\mathrm{T}$ in advanced prostate cancer. Cancer. 115(16): 3670-9.

[50]. Zhou X, Liao Y, Li H, Zhao Z, Liu Q (2016) Dendritic cell vaccination enhances antiangiogenesis induced by endostatin in rat glioma. J Cancer Res Ther. 12(1): 198-203.

[51]. Garg AD, Vandenberk L, Koks C, Tina Verschuere, Louis Boon, et al. (2016) Dendritic cell vaccines based on immunogenic cell death elicit death signals and $\mathrm{T}$ cell-driven rejection of high-grade glioma. SciTransl Med. 8(328).

[52]. Chang CN, Huang YC, Yang DM, Kikuta K, Wei KJ, et al. (2011) A phase I/II clinical trial investigating the adverse and therapeutic effects of a postoperative autologous dendritic cell vaccine in patients with malignant glioma. J ClinNeurosci. 18(8): 1048-54.

[53]. Hunn MK, Bauer E, Wood CE, Gasser O, Dzhelali M, et al. (2015) Dendritic cell vaccination combined with temozolomide retreatment: results of a phase I trial in patients with recurrent glioblastomamultiforme. J Neurooncol. 121(2): 319-29. doi: 10.1007/s11060-014-1635-7.

[54]. Villadangos JA. (2016) Antigen-specific impairment of adoptive T-cell therapy against cancer: players, mechanisms, solutions, and a hypothesis. Immunol Rev. 272(1): 169-82s. doi: 10.1111/imr.12433.

[55]. Everson RG, Antonios JP, Lisiero DN, Soto H, Scharnweber R, et al. (2016) Efficacy of systemic adoptive transfer immunotherapy targeting NY-ESO-1 for glioblastoma. NeuroOncol. 18(3): 368-78. doi: 10.1093/neuoncl nov153.

[56]. Figueroa JA, Reidy A, Mirandola L, Trotter K, Suvorava N, et al., (2015) Chimeric antigen receptor engineering: a right step in the evolution of adoptive cellular immunotherapy. Int Rev Immunol. 34(2):154-87. doi: 10.3109/08830185.2015.1018419.

[57]. Maher J, Brentjens RJ, Gunset G, Rivière I, Sadelain M, et al., (2002) Human T-lymphocyte cytotoxicity and proliferation directed by a single chimeric TCRzeta/CD28 receptor. Nat Biotechnol. 20(1): 70-75.

[58]. Chow KK, Naik S, Kakarla S, Brawley VS, Shaffer DR, et al., (2013) T cells redirected to EphA2 for the immunotherapy of glioblastoma. MolTher. 21(3): 629-37.doi: 10.1038/mt.2012.210.

[59]. Brown CE, Badie B, Barish ME, Weng L, Ostberg JR, et al., (2015) Bioactivity and Safety of IL13R $\alpha 2$-Redirected Chimeric Antigen Receptor CD8+ T Cells in Patients with Recurrent Glioblastoma. Clin Cancer Res. 21(18):4062-72. doi: 10.1158/1078-0432.CCR-15-0428.

[60]. Preusser M, Lim M, Hafler D, Reardon DA, Sampson JH (2015) Prospects of immune checkpoint modulators in the treatment of glioblastoma. Nat Rev Neurol. 11(9): 504-14.

[61]. Topalian SL, Drake CG, Pardoll DM (2015) Immune Checkpoint Blockade: A Common Denominator Approach to Cancer Therapy. Cancer Cell. 27(4): 450-61. doi: 10.1016/j.ccell.2015.03.001.

[62]. Pardoll DM (2012) The blockade of immune checkpoints in cancer immunotherapy. Nat Rev Cancer. 12(4): 252-64 doi: 10.1038/nrc3239.

[63]. Brahmer JR, Drake CG, Wollner I, Powderly JD, Picus J, et al., (2010) Phase I study of single-agent anti-programmed death-1 (MDX-1106) in refractory solid tumors: safety, clinical activity, pharmacodynamics, and immunologic correlates. J Clin Oncol. 28(19): 3167-75.

[64]. Hodi FS, Mihm MC, Soiffer RJ, Haluska FG, Butler M, et al., (2003) Biologic activity of cytotoxic $\mathrm{T}$ lymphocyte-associated antigen 4 antibody blockade in previously vaccinated metastatic melanoma and ovarian carcinoma patients. Proc Natl Acad Sci U S A. 100(8):4712-7.

[65]. Rizvi NA, Mazieres J, Planchard D, Stinchcombe TE, Dy GK, et al., (2015) Activity and safety of nivolumab, an anti-PD-1 immune checkpoint inhibitor, for patients with advanced, refractory squamous non-small-cell lung cancer: a phase II, single-arm trial. Lancet Oncol 16(3): 257-265.

[66]. Yamazaki N, Uhara H, Fukushima S, Uchi H, Shibagaki N, et al., (2015) Phase II study of the immune-checkpoint inhibitor ipilimumab plus dacarbazine in Japanese patients with previously untreated, unresectable or metastatic melanoma. Cancer Chemother Pharmacol 76(5): 969-975.

[67]. Zeng J, See AP, Phallen J, Jackson CM, Belcaid Z, et al., (2013) Anti-PD-1 blockade and stereotactic radiation produce long-term survival in mice with intracranial gliomas. Int J Radiat Oncol Biol Phys 86(2): 343-349.

[68]. Belcaid Z, Phallen JA, Zeng J, See AP, Mathios D, et al., (2014) Focal radiation therapy combined with 4-1BB activation and CTLA-4 blockade yields long-term survival and a protective antigen-specific memory response in a murine glioma model. PLoS One 9(7): e101764.

[69]. Kim JE, Patel MA, Mangraviti A, Kim ES, Theodros D, et al., (2016) Combination therapy with anti-PD-1, anti-TIM-3, and focal radiation results in regression of murine gliomas. Clin Cancer Res 1535.

[70]. Shabo I, Svanvik J. (2011) Expression of macrophage antigens by tumor cells. AdvExp Med Biol. 714: 141-150.

[71]. Zhou W, Ke SQ, Huang Z, Flavahan W, Fang X, et al., (2015) Periostin secreted by glioblastoma stem cells recruits M2 tumour-associated macrophages and promotes malignant growth. Nat Cell Biol 17(2):170-182.

[72]. Parcesepe P, Giordano G, Laudanna C, Febbraro A, Pancione M (2016) Cancer-Associated Immune Resistance and Evasion of Immune Surveillance in Colorectal Cancer. Gastroenterol Res Pract 2016: 6261721.

[73]. Steinert G, Scholch S, Niemietz T, Iwata N, García SA, et al., (2014) Immune escape and survival mechanisms in circulating tumor cells of colorectal cancer. Cancer Res 74(6): 1694-1704.

[74]. Ding P, Wang W, Wang J et al. (2014) Expression of tumor-associated macrophage in progression of human glioma. Cell BiochemBiophys. 70(3):1625-31.

[75]. Nakagawa J, Saio M, Tamakawa N, Yang Z, Xue L (2007) TNF expressed by tumor-associated macrophages, but not microglia, can eliminate glioma. Int J Oncol 30(4): 803-811.

[76]. Liu D, Song L, Wei J, Courtney AN, Gao X, et al., (2012) IL-15 protects NKT cells from inhibition by tumor-associated macrophages and enhances anti-metastatic activity. J Clin Invest 122(6):2221-2233.

[77]. He G, Zhang H, Zhou J, Wang B, Yanhui Chen, et al., (2015) Peritumoral neutrophils negatively regulate adaptive immunity via the PD-L1/ PD-1 signalling pathway in hepatocellular carcinoma. J ExpClin Cancer Res 34: 141.

[78]. Malietzis G, Giacometti M, Kennedy RH, Athanasiou T, Aziz O, et al. (2014) The emerging role of neutrophil to lymphtocyte ratio in determining colorectal cancer treatment outcomes: a systematic review and metaanalysis. Ann SurgOncol. 21(12): 3938-46. 
[79]. Sheng Han, Yang Liu, Qingchang Li, Zhonghua Li, Haipei Hou, et al., (2015) Pre-treatment neutrophil-to-lymphocyte ratio is associated with neutrophil and T-cell infiltration and predicts clinical outcome in patients with glioblastoma. BMC Cancer. 15:617.

[80]. Gianluca FossatiGiovanni RicevutiS. W. EdwardsCarol WalkerAlison Dalton, et al., (1999) Neutrophil infiltration into human gliomas. ActaNeuropathol. 98(4): 349-54.

[81]. Yang L, Liu Z, Wu R, Yao Q, Gu Z, Liu M (2015) Correlation of C-X-C chemokine receptor 2 upregulation with poor prognosis and recurrence in human glioma. Onco Targets Ther. 8: 3203-9.

[82]. Graf MR, Prins RM, Merchant RE (2001) IL-6 secretion by a rat T9 glioma clone induces a neutrophil-dependent antitumor response with resultant cellular, antiglioma immunity. J Immunol. 166(1): 121-9.

[83]. Chu D, Zhao Q, Yu J, Zhang F, Zhang H, et al., (2016) Nanoparticle Targeting of Neutrophils for Improved Cancer Immunotherapy. AdvHealthc Mater. 5(9): 1088-93.

[84]. Fukuhara H, Ino Y, Todo T (2016) Oncolytic virus therapy: A new era of cancer treatment at dawn. Cancer Sci. doi: 10.1111/cas.13027.

[85]. Kaufman HL, Kim DW, DeRaffele G, Mitcham J, Coffin RS, et al., (2010) Local and distant immunity induced by intralesional vaccination with an oncolytic herpes virus encoding GM-CSF in patients with stage IIIc and IV melanoma. Ann SurgOncol. 17(3): 718-30.

[86]. Andtbacka RH, Kaufman HL, Collichio F, et al. (2015) TalimogeneLaherparepvec Improves Durable Response Rate in Patients with Advanced Melanoma. J ClinOncol. 33(25): 2780-8.

[87]. Andtbacka RH, Ross M, Puzanov I, Milhem M, Collichio F, et al., (2016) Patterns of Clinical Response with TalimogeneLaherparepvec (T-VEC) in Patients with Melanoma Treated in the OPTiM Phase III Clinical Trial. Ann SurgOncol.
[88]. Patel D, Foreman P, Nabors B, Riley K, Gillespie Y, et al., (2016) Design of a Phase I Clinical Trial to Evaluate M032, a Genetically Engineered HSV-1 Expressing IL-12, in Patients with Recurrent/Progressive GlioblastomaMultiforme, Anaplastic Astrocytoma, or Gliosarcoma. Hum Gene TherClin Dev. 27(2): 69-78.

[89]. Cobbs CS, Harkins L, Samanta M, Gillespie GY, Bharara S, et al., (2002) Human cytomegalovirus infection and expression in human malignant glioma. Cancer Res. 62(12): 3347-50.

[90]. Schuessler A, Smith C, Beagley L, Boyle GM, Rehan S, et al., (2014) Autologous T-cell therapy for cytomegalovirus as a consolidative treatment for recurrent glioblastoma. Cancer Res. 74(13): 3466-76.

[91]. CMV-specific Cytotoxic T Lymphocytes Expressing CAR Targeting HER2 in Patients with GBM (HERT-GBM), ClinicalTrials.gov, 2016.

[92]. Liu X, Barrett DM, Jiang S, Fang C, Kalos M, et al., (2016) Improved anti-leukemia activities of adoptively transferred $\mathrm{T}$ cells expressing bispecific T-cell engager in mice. Blood Cancer J. 6(6):e430.

[93]. Johnson LA, Scholler J, Ohkuri T, Kosaka A, Patel PR, et al., (2015) Rational development and characterization of humanized anti-EGFR variant III chimeric antigen receptor T cells for glioblastoma. SciTransl Med. 7(275).

[94]. Patel MA, Kim JE, Theodros D, Tam A, Velarde E, et al., (2016) Agonist anti-GITR monoclonal antibody and stereotactic radiation induce immunemediated survival advantage in murine intracranial glioma. J Immunother Cancer. 4:28.

[95]. Mantovani A, Schioppa T, Porta C, Allavena P, Sica A (2006) Role of tumorassociated macrophages in tumor progression and invasion. Cancer Metastasis Rev. 25(3): 315-22.

[96]. Scheurer ME, Bondy ML, Aldape KD, Albrecht T, El-Zein R (2008) Detection of human cytomegalovirus in different histological types of gliomas. ActaNeuropathol. 116(1): 79-86. 